\title{
Elucidation of the FKBP25-60S Ribosomal Protein L7a Stress Response Signaling During Ischemic Injury
}

\author{
Quan Jiang $^{\mathrm{b}}$ Gang Wu ${ }^{\mathrm{a}, \mathrm{b}}$ Lin-Yanga,b Ya-Ping Lu ${ }^{\mathrm{a}, \mathrm{b}}$ Xiu-Xiu Liu ${ }^{\mathrm{a}, \mathrm{b}}$ Feng Han ${ }^{\mathrm{b}, \mathrm{c}}$ \\ Ya-Ping Deng ${ }^{d} \quad$ Xu-Chun Fu ${ }^{a}$ Qi-Bing Liue Ying-Mei Lu
}

aSchool of Medicine, Zhejiang University City College, Hangzhou, Zhejiang, 'DInstitute of Pharmacology and Toxicology, College of Pharmaceutical Sciences, Zhejiang University, Hangzhou, cKey Laboratory of Cardiovascular \& Cerebrovascular Medicine, School of Pharmacy, Nanjing Medical University, Nanjing, 'Department of Pharmacy, Zhejiang Xiaoshan Hospital, Hangzhou, Zhejiang, 'Department of pharmacology, School of basic medicine and life science. Hainan medical college, Haikou, China

\section{Key Words}

Fkbp25 • 60S ribosomal protein L7a • Nitrosative stress • Nuclear translocation • Oxygenglucose deprivation

\begin{abstract}
Background/Aims: Peptidyl-prolyl cis-trans isomerase FKBP25 is a member of the FK506binding proteins family which has peptidyl-prolyl cis/trans isomerase domain. The biological function and pathophysiologic role of FKBP25 remain elusive. Methods: The spatio-temporal changes in expression of endothelial FKBP25 upon oxygen-glucose deprivation (OGD) treatment were examined by Western blot and immunofluorescence. The immunoprecipitation and fluorescence resonance energy transfer (FRET) were used to address the interacting proteins with FKBP25. Results: In the present study, nuclear translocation of FKBP25 was observed following OGD in cultured endothelial cells. Intriguingly, FKBP25 nuclear translocation was further validated in peroxynitrite (ONOO-)-treated endothelial cells. Coimmunoprecipitation and FRET data indicated that FKBP25 translocated into the nucleus, in which it interacted with 60 S ribosomal protein $\mathrm{L} 7 \mathrm{a}$, while overexpression FKBP25 protect endothelial cells against OGD injury. Conclusion: Our findings reveal that the nuclear import of FKBP25 and binding with $60 \mathrm{~S}$ ribosomal protein $\mathrm{L7a}$ are protective stress responses to ischemia/nitrosaive stress injury.

(C) 2018 The Author(s)

Published by S. Karger AG, Basel
\end{abstract}

\section{Introduction}

Peptidyl-prolyl cis-trans isomerase FKBP25 is a member of the FK506-binding proteins family which has peptidyl-prolyl cis/trans isomerase domain [1-3]. Proline is the only amino

Quan Jiang and Gang Wu contributed equally to this work.

Qi-Bing Liu

and Ying-Mei Lu
Department of Pharmacology, School of Basic Medicine and Life Science Hainan

Medical College, Haikou, (China)

Tel. +86-898-31350701, E-Mail hy0207043@hainmc.edu.cn / lufx@zju.edu.cn 
acid of the 20 basic amino acids that provides the possibility for protein conformation change with cis/trans isomerization. Previous work has shown that peptidyl-prolyl cis-trans isomerase is involved in many critical biochemical processes, including protein transcription, folding, signal transduction, etc. [4-6].

Stroke is a serious disease that is hazardous human health and has high morbidity, disability and mortality rates. It remains an important question to explore the molecular events and drug targets in neurovascular units following brain ischemia. Notably, FK506 is considered as a powerful neuroprotective agent in focal and global cerebral ischemia [7, 8]. FK506 binds to intracellular proteins (FKBP), which elicits the peptidyl-prolyl cis/trans isomerase activity [9]. FKBP12 is involved in the neuroprotective effects of FK506 in an ischemic cortical cell culture model [10], FKBP51-PHLPP2-AKT signaling complex plays a critical role in mediating cell death in cerebral ischemia/reperfusion injury in rats [11] and FKBP38 also mediates FK506 neuroprotective and neurotrophic properties in brain ischemia [12]. Recent research reveals that FKBP25 can bind with DNA and RNA, regulate microtubule polymerization to modulate cell cycle progression, and participate in ribosome biogenesis [2, 3, 13-15]. And the research by Lopez E et al. showed FKBP25 regulate TRPC6 to control non-capacitative calcium entry in HEK-293 cells [16]. However, the pathophysiologic role of FKBP25 in brain diseases remains largely unknown.

In the present study, we sought to determine the spatiotemporal changes in FKBP25 protein levels during ischemic injury. Our data demonstrate a protective role of the nuclear accumulation of FKBP25 following stroke and identify 60S ribosomal protein L7a as a key regulator of endothelial intracellular FKBP25 trafficking during ischemic injury.

\section{Materials and Methods}

\section{Reagents}

RPMI 1640 Medium (1640), Dulbecco's Modified Eagle's Medium (DMEM) and fetal bovine serum (FBS) were purchased from Gibco (Carlsbad, CA, USA). Alexa Fluor 488-conjugated anti-mouse IgG, Alexa Fluor 594-conjugated anti-rabbit IgG were obtained from Invitrogen (Carlsbad, CA, USA). Peroxynitrite (ONOO) synthesized from isoamyl nitrite and hydrogen peroxide was purchased from Millipore (Merck KGaA, Darmstadt, Germany). Unless otherwise stated, all reagents and chemicals were obtained from Sigma-Aldrich (St. Louis, MO, USA) [17].

\section{Cell culture}

Cells were cultured in DMEM supplemented with 10\% FBS, 100 units $/ \mathrm{ml}$ penicillin and $100 \mathrm{mg} / \mathrm{ml}$ streptomycin except HBMEC which cultured in 1640 supplemented with 10\% FBS [18]. EA.hy926 cells, Human Umbilical Vein Endothelial Cells (HUVEC) and HEK-293 cells were purchased from ATCC (Manassas, VA, USA). Human Brain Microvascular Endothelial Cells (HBMECs) were purchased from ScienCell Research Laboratories (Carlsbad, CA, USA).

\section{Mice}

The wild-type (C57BL/6 strain) were housed under standard conditions with a 12-h light/dark cycle and had free access to food and water ad libitum. 8 to 12-week-old male mice and the weight of the mice between 22-25 g were used in the study. All animal use procedures were approved by the Committees at Zhejiang University.

Oxygen-glucose deprivation (OGD) exposure

Oxygen-glucose deprivation (OGD) was performed as we previously reported [19]. Briefly, at a density of $80-90 \%$, the cultured cells were washed with glucose-free Hank's balanced salt solution (HBSS) three times and cultured with HBSS for the following experiments. Then the cells were placed in an airtight experimental hypoxia chamber (Billups-Rothenberg, San Diego, CA, USA) and removed the internal air by ejecting with a gas mixture composed of $95 \% \mathrm{~N}_{2}$ and $5 \% \mathrm{CO}_{2}$ for $5 \mathrm{~min}$ [19]. 


\section{Cellular Physiology Cell Physiol Biochem 2018;47:2018-2030 \begin{tabular}{l|l|l} 
and Biochemistry & $\begin{array}{l}\text { DOI: 10.1159/000491470 } \\
\text { Published online: July 03, } 2018\end{array}$ & $\begin{array}{l}\text { C) } 2018 \text { The Author(s). Published by S. Karger AG, Basel } \\
\text { www.karger.com/cpb }\end{array}$
\end{tabular}}

\section{Plasmid constructs and transfections}

Total RNA was isolated using TRIzol (Takara, Tokyo, Japan) from EA.hy926 according to the manufacturer's protocol and total RNA was reverse transcribed to cDNA by RT-PCR. The DNA of FKBP25 was obtained from cDNA by PCR amplification. Full length and truncated fragment of FKBP25 were inserted into a blank plasmid vector encoding EGFP. FKBP25 was modified by PCR and subcloned into pEYFP-C1 (Clontech). 60S ribosomal protein L7a (RPL7) was subcloned into pECFP-C1 (Clontech). All constructs were confirmed by sequencing and transfected by using Lipofectamine 3000 (Invitrogen, Carlsbad, CA, USA) when the cell density approached $60-70 \%$. The culture medium was replaced six hours later with $10 \%$ fetal bovine serum, and the cells were cultured for another two days. For fluorescence resonance energy transfer (FRET), HEK-293 cells were transiently co-transfected with EYFP-FKBP25 and ECFP-RPL7.

\section{Image acquisition}

Cells were cultured in glass-bottomed dishes and infected with lentivirus encoding FKBP25-EGFP. Live imaging experiments were performed on an epifluorescence microscope (Olympus, DU-897D-CS0) using a $60 \times$ oil immersible objective and a CCD camera (ANDOR iXon3). ONOO- $(250 \mu \mathrm{M})$ was added to the culture medium before image acquisition. Images were collected with laser at $\lambda_{\text {ex }} 488 \mathrm{~nm}$ for $60 \mathrm{~min}$ [20].

\section{Cell fractionation and immunoblotting analysis}

Treated cells were washed in cold PBS before collected and homogenized by buffer A containing 0.25 $\mathrm{mM}$ sucrose, $1 \mathrm{mM}$ EDTA-free acid, $15 \mathrm{mM}$ Tris- $\mathrm{HCl}$ (pH 7.5) and protease inhibitors. Then centrifuge with $1000 \mathrm{~g}$ for $10 \mathrm{~min}$ to get the supernatant which is the cytosol protein. Cells pellets were further washed with buffer A twice to remove residual cytosolic proteins. The purified nuclei were resuspended with buffer C containing $50 \mathrm{mM}$ Tris-HCl (pH 7.4), 4 mM EGTA, $10 \mathrm{mM}$ EDTA, 0.5\% Triton X-100, $0.5 \mathrm{M} \mathrm{NaCl}, 50 \mathrm{mM}$ $\mathrm{NaF}, 30 \mathrm{mM}$ sodium pyrophosphate, $1 \mathrm{mM} \mathrm{Na}_{3} \mathrm{VO}_{4}$ and protease inhibitors [19]. The immunoblotting was carried out by sodium dodecyl sulfate-polyacrylamide gel electrophoresis (SDS-PAGE) after determination of protein concentrations using Bradford's solution [21, 22]. FKBP25 (1:1000; Santa Cruz Biotechnology, Dallas, Texas, USA), $\beta$-actin (1:5000; Sigma Aldrich, St. Louis, MO, USA), lamin B1 (1:1000; Santa Cruz Biotechnology, Dallas, Texas, USA) primary antibodies were used, followed by a secondary antibody used for detection. Protein expression were analyzed by Image J software (NIH, Bethesda, Maryland, USA), and samples immunoblotted for $\beta$-actin and lamin B1 as cytoplasmic and nuclear markers, respectively.

\section{Confocal immunofluorescence staining}

Cells were grown on glass coverslips in a 24 well plate and fixed in $4 \%$ formaldehyde/PBS after indicated treatment for immunofluorescence analysis as previously reported [23]. Cells were fixed in a formaldehyde/ sucrose solution for $10 \mathrm{~min}$ and then permeabilized in $0.1 \%$ Triton-X solution. Cells were immunolabeled with FKBP25 (1:200, Santa Cruz Biotechnology, Santa Cruz, Dallas, Texas, USA) and HSP27 (1:200, Cell Signaling Technology, Danvers, MA, USA) at $4^{\circ} \mathrm{C}$ for $24 \mathrm{~h}$. Coverslips were washed and incubated with Alexa Fluor 488-conjugated and Alexa Fluor 594-conjugated second antibody for $4 \mathrm{~h}$ at room temperature. 4, 6-diamidino-2-phenylindole dihydrochloride bisbenzimide (DAPI, $5 \mu \mathrm{mol} / \mathrm{L}$ ) were used to stain nuclei. The imaging were visualized by confocal microscopy (Nikon A1R, Nikon, Tokyo, Japan).

\section{Immunoprecipitation and silver staining}

The immunoprecipitation was conducted with whole-cell extracts with or without OGD treatment. The lysis buffer containing $10 \mathrm{mM}$ Tris- $\mathrm{HCl}$ (pH 7.5), 0.5\% NP-40, $1 \mathrm{mM}$ EDTA, $150 \mathrm{mM} \mathrm{NaCl}$ and supplemented with protease inhibitor. The protein extracts were incubated overnight with the appropriate antibodies plus protein A/G beads. Immunoprecipitated proteins were electrophoresed by SDS-PAGE and obtained gel for silver staining (Bio-Rad, Hercules, CA, USA). The visible band was cut and digested with trypsin and then subjected to liquid chromatography (LC) MS/MS analysis [24].

\section{Fluorescence Resonance Energy Transfer}

Fluorescence resonance energy transfer (FRET) was examined using the acceptor photobleaching method as previous describes [18]. Briefly, EYFP-labeled FKBP25 (EYFP-FKBP25) and ECFP-labeled RPL7 (ECFP-RPL7) were co-transfected into HEK293 for FRET analysis after OGD exposure. Images were acquired with a confocal microscope equipped with a $\times 60$ oil-immersion objective. The ECFP fluorophore (donor) 
was excited with $405 \mathrm{~nm}$ line of argon laser and an EYFP fluorophore (acceptor) with a $515 \mathrm{~nm}$ line. The cells were bleached with EYFP channel by scanning a region of interest (ROI) using the $515 \mathrm{~nm}$ laser line at $100 \%$ intensity, ECFP images were acquired to evaluate changes in donor fluorescence before and after EYFP photobleaching.

\section{Statistical analysis}

Statistical significance for differences among groups was tested by one-way analysis of variance, followed by post-hoc Tukey tests between control and other groups. Asterisks $\left({ }^{*} P<0.05,{ }^{* *} P<0.01,{ }^{* * *} P<\right.$ 0.001 ,) denote statistical significance in graphs. All data are expressed as the mean \pm S.E.M.

\section{Results}

FKBP25 protein translocated from the cytosol to the nucleus in endothelial cells following $O G D$

To explore the pathophysiologic role of FKBP25 in ischemia in vitro, we first determined the temporal changes of FKBP25 over 1 to $24 \mathrm{~h}$ in EA.hy926 endothelial cells under OGD treatments. As shown in Fig. 1, cytosolic FKBP25 protein levels were decreased in a time-dependent manner following OGD (Fig. 1A and B), whereas its protein levels were significantly elevated in the nucleus (Fig. 1C and D). There was a similar increasing pattern of nuclear protein levels of FKBP25 in human umbilical vein endothelial cells (HUVEC) (Fig. 2A and B). Consistently, nuclear translocation of FKBP25 was also observed in human brain microvessel endothelial cells (HBMEC) (Fig. 2C and D). Furthermore, the expression of endogenous FKBP25 in endothelial cells-enriched tissue is high (Fig. 2E and F). Together, FKBP25 is a sensitive protein that translocate into the nucleus in endothelial cells after OGD injury.

\footnotetext{
Subcellular distribution of endothelial FKBP25 following $O G D$

Nuclear translocation and activation of molecules during ischemia might play a role in conveying signals from the cytosol to the nucleus [19]. Here, we further validate whether OGD exposure triggers FKBP25 nuclear translocation by immunofluorescence staining. Activation of heat shock protein 27 (HSP27) is a hallmark of ischemic injury [17]. Double staining of FKBP25 and HSP27 in EA.hy926 endothelial cells showed that the FKBP25 protein was mainly localized in the cytosol, whereas increased protein levels of FKBP25 were detected in the nucleus after OGD treatment (Fig. 3A). A similar result was observed in HUVECs (Fig. 3B). Thus, FKBP25 is a sensitive response molecule in ischemia-induced endothelial damage in vitro.
}

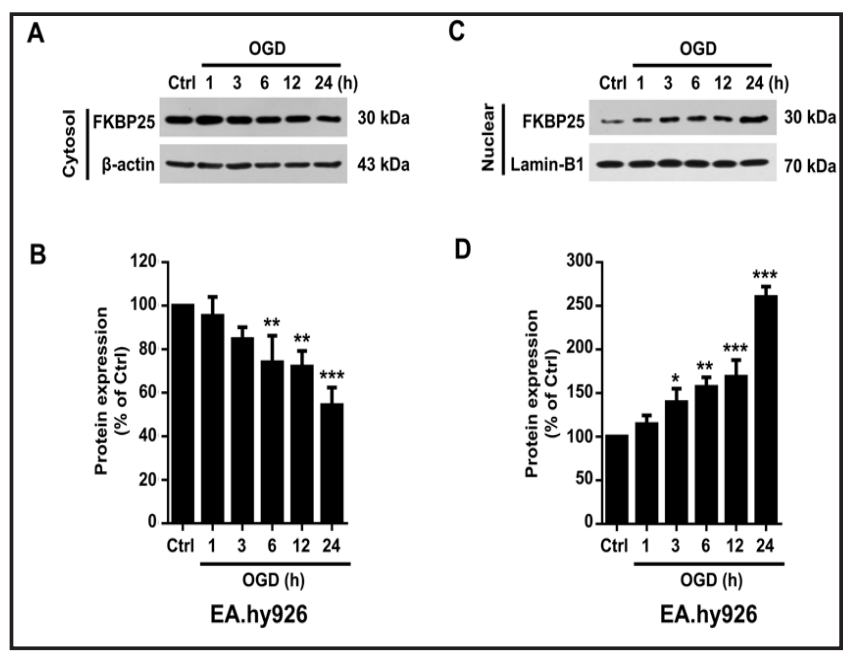

Fig. 1. Subcellular localization of FKBP25 in EA.hy926 cells during OGD exposure. (A) Temporal changes in cytosolic FKBP25 levels in EA.hy926 cells during OGD. (B) Quantitative analyses of the results as shown in (A) and presented in the bar graph. ${ }^{* *} \mathrm{P}<0.01,{ }^{* * *} \mathrm{P}<0.001$ versus control; Ctrl, control. (C) Nuclear translocation of FKBP25 in EA.hy926 cells following OGD. (D) Quantitative analyses of the results as shown in (C) and presented in the bar graph. ${ }^{*} \mathrm{P}<0.05,{ }^{* *} \mathrm{P}<0.01,{ }^{* * *} \mathrm{P}<0.001$ versus control; Ctrl, control. Data are expressed as means \pm S.E.M of at three independent experiments. 
$\begin{array}{lr}\text { Spatiotemporal } & \text { changes } \\ \text { of FKBP25 nuclear } \\ \text { translocation } \\ \text { nitrosative stress } \\ \text { Oxidant pen } \\ \end{array}$ (ONOO) is formed by the reaction of superoxide $\left(\mathrm{O}_{2}{ }^{-}\right)$ and nitric oxide (NO), which could be generated by OGD [17]. ONOO is a highly reactive oxidant involved in modulating signaling pathway via attacking and inactivating many proteins via nitration to tyrosine residues [17-19]. To investigate whether FKBP25 translocation may be a consequence of nitrosative stress biochemically, protein extracts from cells treated with ONOO $^{-}$were prepared, and nuclear and cytoplasmic fractions were analysed on Western blots. Increased levels of nuclear FKBP25 were detected in a concentration dependent way after $\mathrm{ONOO}^{-}$ treatment (Fig. 4A and B). To follow the translocation of FKBP25 to the nucleus after nitrosative stress, we monitored dynamic changes of FKBP25EGFP for 60 min with or without ONOO- $(250 \mu \mathrm{M})$ treatment (Fig. $4 \mathrm{C}$ and D). Live cell fluorescence image analysis indicated a cytoplasmic localization of

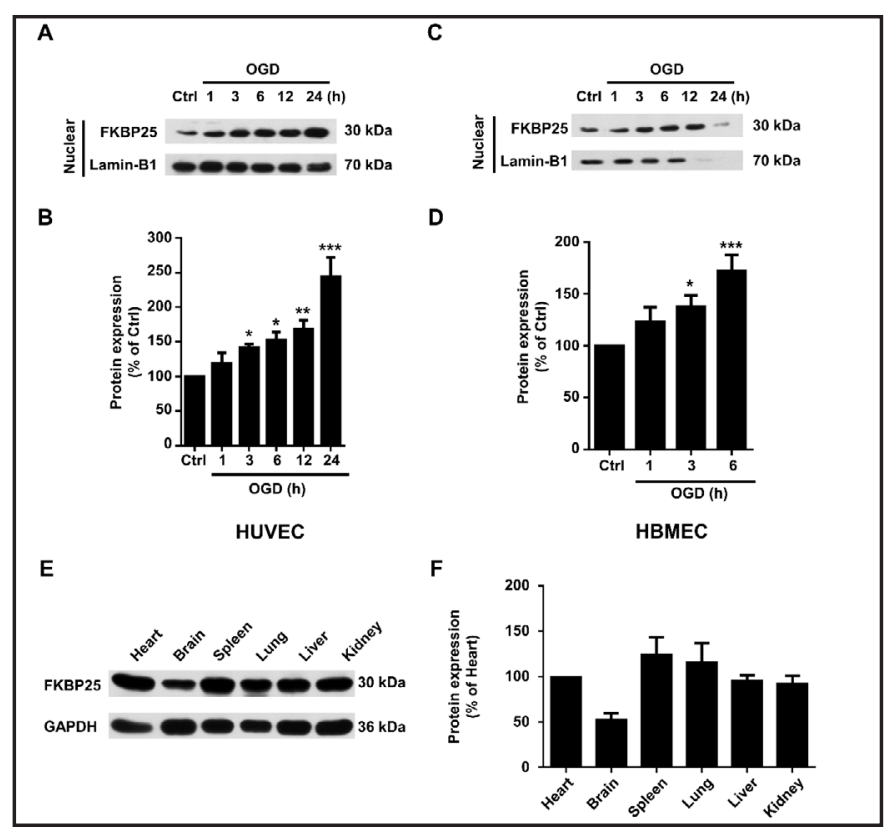

Fig. 2. Subcellular localization of FKBP25 in HUVEC and HBMEC during OGD and the expression of FKBP25 in the different organs of mice. (A) Nuclear translocation of FKBP25 in HUVEC following OGD. (B) Quantitative analyses of the results as shown in (A) and presented in the bar graph. ${ }^{* *} \mathrm{P}<0.01,{ }^{* * *} \mathrm{P}<0.001$ versus control; Ctrl, control. (C) Nuclear translocation of FKBP25 in HBMEC following OGD. (D) Quantitative analyses of the results as shown in (C) and presented in the bar graph. ${ }^{*} \mathrm{P}<0.05,{ }^{* * *} \mathrm{P}<0.001$ versus control; Ctrl, control. Data are expressed as means \pm SEM of at three independent experiments. (E) Extracts of protein from heart, brain, spleen, lung, liver and kidney in C57BL/6 mice. The protein levels of FKBP25 were detected by Western blot. (F) Quantitative analyses of the results presented in the bar graph. $n=4$ mice for each group.

FKBP25-EGFP in control cells, whereas translocation of FKBP25-EGFP from the cytoplasm to the nucleus increased in a time-dependent manner following $\mathrm{ONOO}^{-}$exposure (Fig. 4C and D). Taken together, these results indicate that the increased level of FKBP25 in the nucleus after OGD was a consequence of nitrosative stress, which directly correlated with the concentration of $\mathrm{ONOO}$.

\section{The nuclear location of FKBP25 independent the PPIase domain}

To investigate the mechanism of FKBP25 nuclear translocation. We immunoprecipitated FKBP25 from endothelial cells using antibodies directed against the protein and subjected the immunoprecipitates to Western blot and mass spectrometry analysis (Fig. 5A). We found that ribosomal protein L7a (RPL7) was selectively enriched in the nuclear FKBP25 co-immunoprecipitates of endothelial cells following OGD compared with the control cells. Indeed, RPL7 is a component of the 60S large ribosomal subunit and has important functions involved in ribosome assembly and pre-rRNA processing [25].

It has been determined that FKBP25 consists of a conserved FK506/rapamycinbingding domain (FKBD) and a strongly hydrophilic N-terminal domain which has a helixloop-helix motif (HLH) [3] (Fig. 5B). To ascertain the intracellular localization of FKBP25 and to determine which domains of FKBP25 are necessary for nuclear import, we generated 


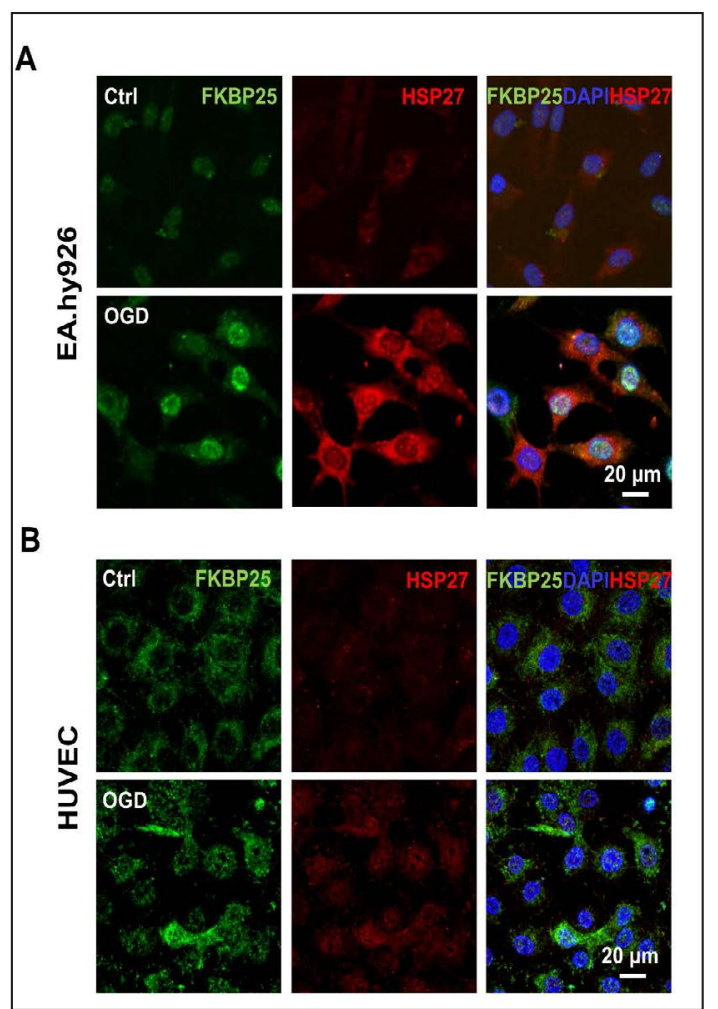

Fig. 3. Confocal imaging of FKBP25 nuclear translocation upon OGD. (A) Representative immunofluorescence images of FKBP25 (green) and HSP27 (red) are indicated in EA.hy926 after OGD treatment. (B) Subcellular localization of FKBP25 (green) detected by laser confocal microscopy. The immunofluorescence image of FKBP25 (green) and HSP27 (red) were examined in the HUVEC after OGD treatment. DAPI counterstaining (blue) indicates nuclear localization. Scale bar $=20 \mu \mathrm{m}$. Ctrl, control.

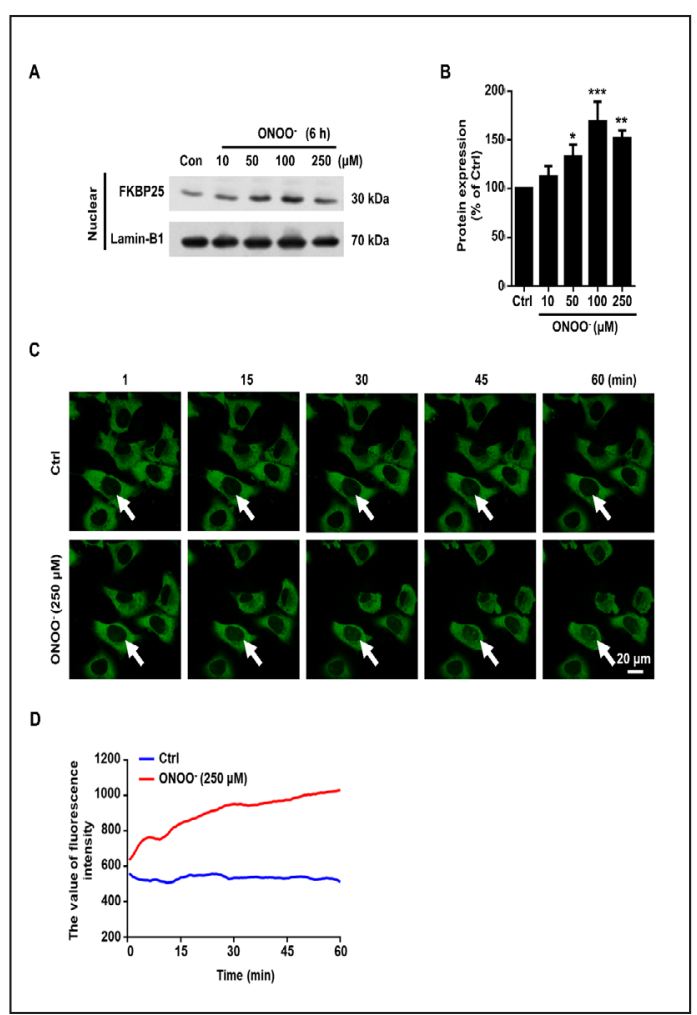

Fig. 4. Nitrosative stress triggered FKBP25 translocation from cytosol to the nucleus. (A) Immunoblot analysis of nuclear extracts of EA.hy 926 cells incubated with indicated concentrations of $\mathrm{ONOO}^{-}$for 6 h. (B) Quantification of ONOO- induced FKBP25 translocation as shown in (A). Data were from three independent experiments and expressed as the means \pm SEM. ${ }^{*} \mathrm{P}<0.05,{ }^{* *} \mathrm{P}<0.01,{ }^{* *} \mathrm{P}<0.001$ versus control; Ctrl, control. (C) Living cells recording at indicated time points of EA.hy926 infected with lentivirus FKBP25-EGFP treated with or without ONOO- as indicated. (D) Quantitative analysis of FKBP25-EGFP fluorescence value localized in nucleus as shown in (C).

either EGFP-tagged full-length FKBP25 (homo sapiens) or deletion mutants ( $\Delta$-1-108-EGFP or $\Delta$-109-224-EGFP). Confocal microscopy imaging indicated that EGFP expressions with the truncated FKBP25 ( $\Delta$-1-108-EGFP) was mainly detectable in the cytosolic compartment, as did full-length FKBP25-EGFP (Fig. 5C). Surprisingly, EGFP expression was principally detected in the nuclear compartment following truncated FKBP25 $(\Delta-109-224-\mathrm{EGFP})$ (N-terminal domain deprivation) transfection (Fig. 5C). These data indicate that the nuclear localization of FKBP25 is independent of the first 108 residues of the coding sequence of FKBP25, which play a critical role in modifying FKBP25 subcellular localization.

Then, we tested whether RPL7 might affect FKBP25 subcellular localization in cells. We transfected HEK-293 cells with ECFP-tagged RPL7 and FKBP25-EGFP (1-108 or 109-224 or full-length) (Fig. 5D). The cells were transfected with FKBP25-EGFP (1-108) or FKBP25-EGFP (full-length) were both diffusely localized in the cytoplasm. Interestingly, EGFP expression was localized in the nucleus from cytosol when co-transfected with ECFP-RPL7 and EGFP- 


\section{Cellular Physiology and Biochemistry}

Cell Physiol Biochem 2018;47:2018-2030

\begin{tabular}{l|l}
\hline DOI: $10.1159 / 000491470$ & (C) 2018 The Author(s). Published by S. Karger AG, Basel
\end{tabular}

Jiang et al.: FKBP25 Chaperone the 60S Ribosomal Protein L7a tagged full-length human FKBP25 or deletion mutants $(\Delta-1$-108-EGFP) in HEK-293 cell. Intensity analysis of the line region (Fig. 5D) in HEK-293 cells revealed that immunofluorescence signals of the two proteins overlap at the nucleus (Fig. 5E). Morever, the FKBP25-EGFP (1-108) and FKBP25-EGFP fluorescence in nucleus was decreased in cells while co-transfected with ECFP-labelled RPL7 constructs under rapamycin treatment. However, FK506 treatment did not affect the cytosol-tonucleus translocation (Fig. 6). These results indicate that expression of RPL7 can lead to a dramatic change in FKBP25 subcellular localization, and that this effect is N-terminal domain-dependent.

$\begin{array}{lr}\text { FRET analysis of } \\ \text { interaction } & \text { between } \\ \text { FKBP25 and } & \text { RPL7 } \\ \text { following OGD }\end{array}$

To monitor a direct molecular interaction between FKBP25 and RPL7 in living cells, acceptor photobleaching FRET analysis was performed between EYFP-FKBP (acceptor) and ECFP-RPL7 (donor) using endothelial cells expressing these two proteins (Fig. 7A). The acceptor fluorescence was collected to assess ECFP images under EYFP photobleaching.

We observed that EYFP distributed in cytosol and unchanged in ECFP fluorescence after EYFP bleaching in control (Fig. 7B). However, under OGD treatments, EYFP and ECFP fluorescence appeared both in cytosol and nucleus in endothelial cells. Interestingly, CFP emission becomes stronger in the cytosol of OGD-

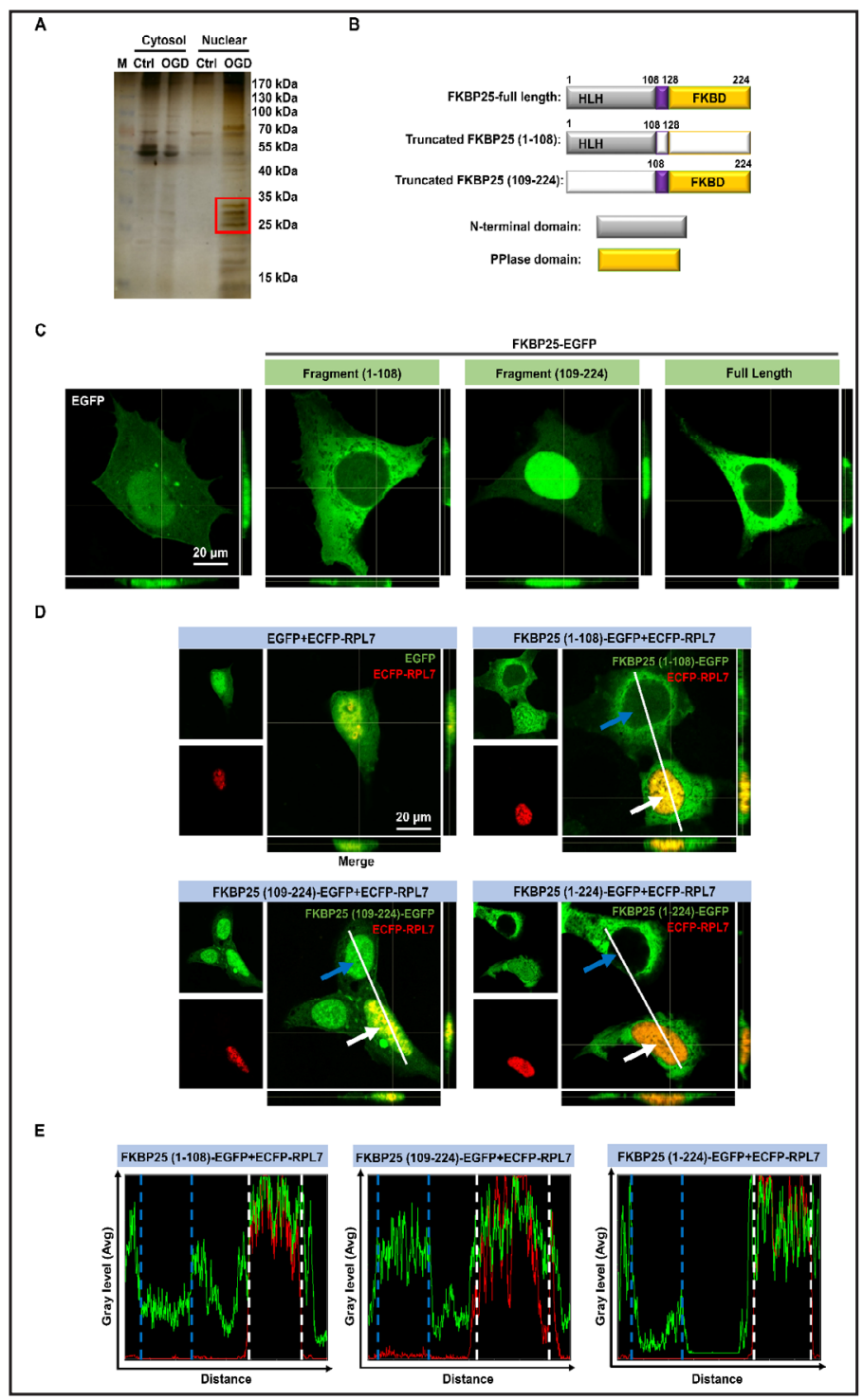

Fig. 5. FKBP25 cytosol distribution was dependent on N-terminal of 1-108 amino acid residues and changed into nuclear by RPL7. (A) The silver staining of control and OGD-treated EA.hy926 endothelial cells from cytosol and nuclear protein with immunoprecipitation. (B) Schematic diagram of the truncated mutants from FKBP25. (C) The representative confocal microscopy images of FKBP25-EGFP, Truncated FKBP25-EGFP (1-108) and FKBP25-EGFP (109-224) in EA.hy926 cells. Scale bar $=20 \mu \mathrm{m}$. (D) Constructs encoding ECFPRPL7 and FKBP25-EGFP, FKBP25-EGFP (1-108) or FKBP25-EGFP (109-224) were transfected into HEK-293 cells and visualized using confocal microscopy. Blue arrow showed the cells only transfected with EGFP-labeled constructs. White arrow showed the cells transfected with EGFP-labeled and ECFP-labeled constructs. White line indicated the region chosen for a line profile of fluorescence intensities. Scale bar $=20 \mu \mathrm{m}$. (E) Line plots of staining intensity along the white line shown in (D) that was drawn across the cell, and reveal that RPL7 induced cytosol FKBP25 translocate into nucleus. 


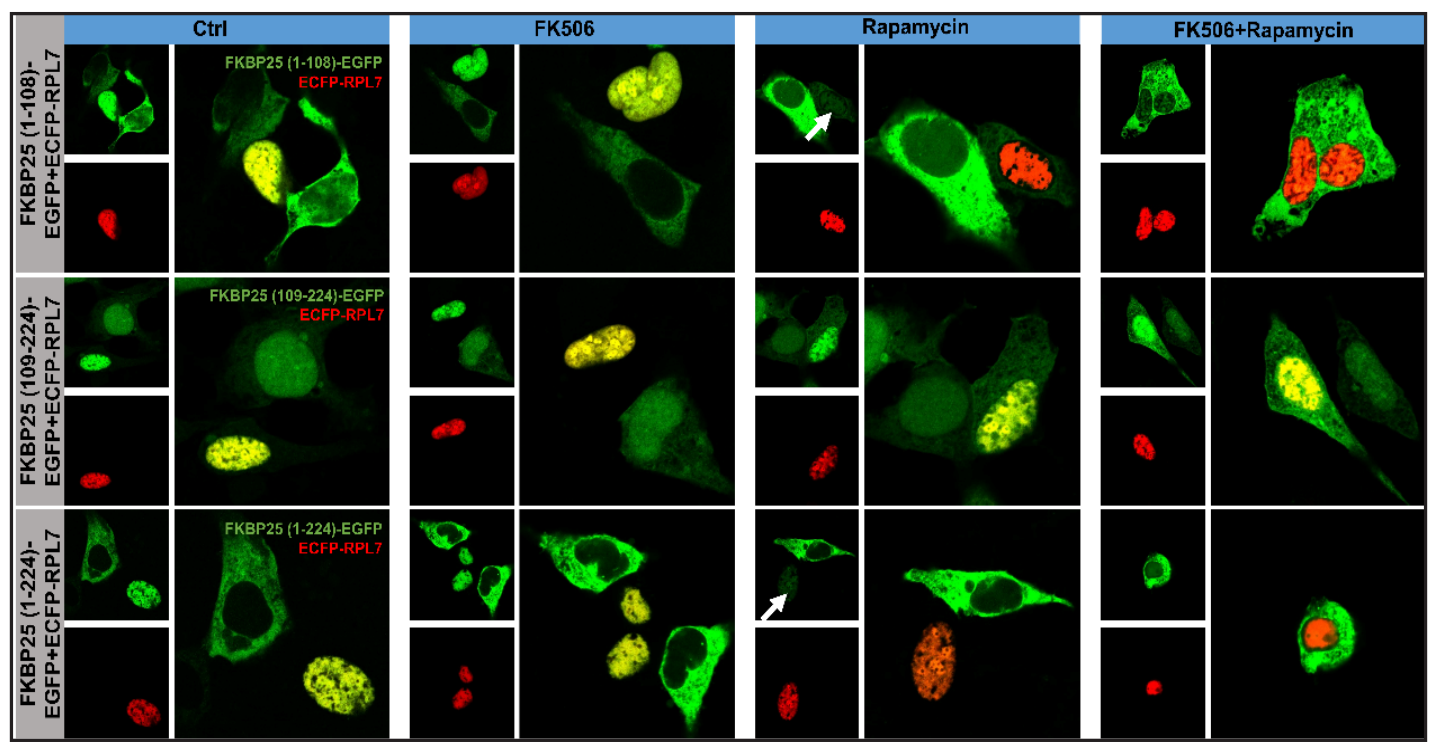

Fig. 6. Effect of rapamycin and FK506 on FKBP25 subcellular localization. The FKBP25-EGFP, FKBP25-EGFP (1-108) or FKBP25-EGFP (109-224) with ECFP-RPL7 were co-transfected into HEK-293 cells. The confocal imaging were captured following incubation with FK506 $(2 \mu \mathrm{M})$ and/or rapamycin $(2 \mu \mathrm{M})$ for $6 \mathrm{~h}$. The FKBP25-EGFP (1-108) and FKBP25-EGFP fluorescence (White arrow) in nucleus was decreased in cells while co-transfected with ECFP-labeled RPL7 constructs under rapamycin treatment.

treated endothelial cells after photobleaching YFP using a $515 \mathrm{~nm}$ laser (Fig. 7C and D). As illustrated in Fig. 7E, when the CFP molecule is excited by a laser at $458 \mathrm{~nm}$, both CFP and YFP fluoresce if these two molecules are close enough to each other for FRET to occur. As a result, OGD treatments strengthened the interaction between RPL7 and FKBP25 (Fig. 7E).

To evaluate the pathophysiological role of FKBP25 nuclear translocation during ischemia, we determined the apoptosis inhibiting capacity of full length FKBP25 in endothelial cells. As shown in Fig. 8A and 8B, the cleavage of poly ADP ribose polymerase 1 (PARP-1) was significant increased $(169.00 \pm 11.37)$ in endothelial cells upon OGD treatment. Overexpression of FKBP25 (full length) significantly inhibited PARP-1 cleavage $(129.30 \pm 8.09)$. Therefore, our data indicating the anti-apoptotic effect of FKBP25 nuclear translocation during ischemia.

\section{Discussion}

FK506 is an immunosuppressant and shows promise for treating ischemic brain damage $[8,9]$. FKBPs, as FK506-binding proteins, have in common peptidyl-prolyl cis/trans isomerase activity $[4,5]$, but the precise role in ischemia damage remains largely unknown. Here, we found that FKBP25 translocated from the cytosol to nucleus under OGD and ONOO- treatment in endothelial cells, which is independent of the first 108 residues of the coding sequence of FKBP25. We further identified that 60S ribosomal protein L7a interact with FKBP25 which maybe mediate FKBP25 nuclear translocation. Moreover, we found that nuclear FKBP25 overexpression decreased PARP1 protein level. Therefore, these observations reveal new biological activities of FKBP25 and indicate that the nuclear translocation of FKBP25 may mediate protection against endothelial cells during ischemia injury.

Endothelial cells are sensitive pathological events in early stage of cerebral ischemia, which elicit critical impact on barrier stability $[17,19,26,27]$. Here we found OGD insult of endothelial cells led to continuous elevation of FKBP25 nuclear protein levels at least until $24 \mathrm{~h}$ after treatments. However, the molecular events governing the translocation of FKBP25 remain unclear. Accumulating evidences indicating that nitrosative stress participate in brain 


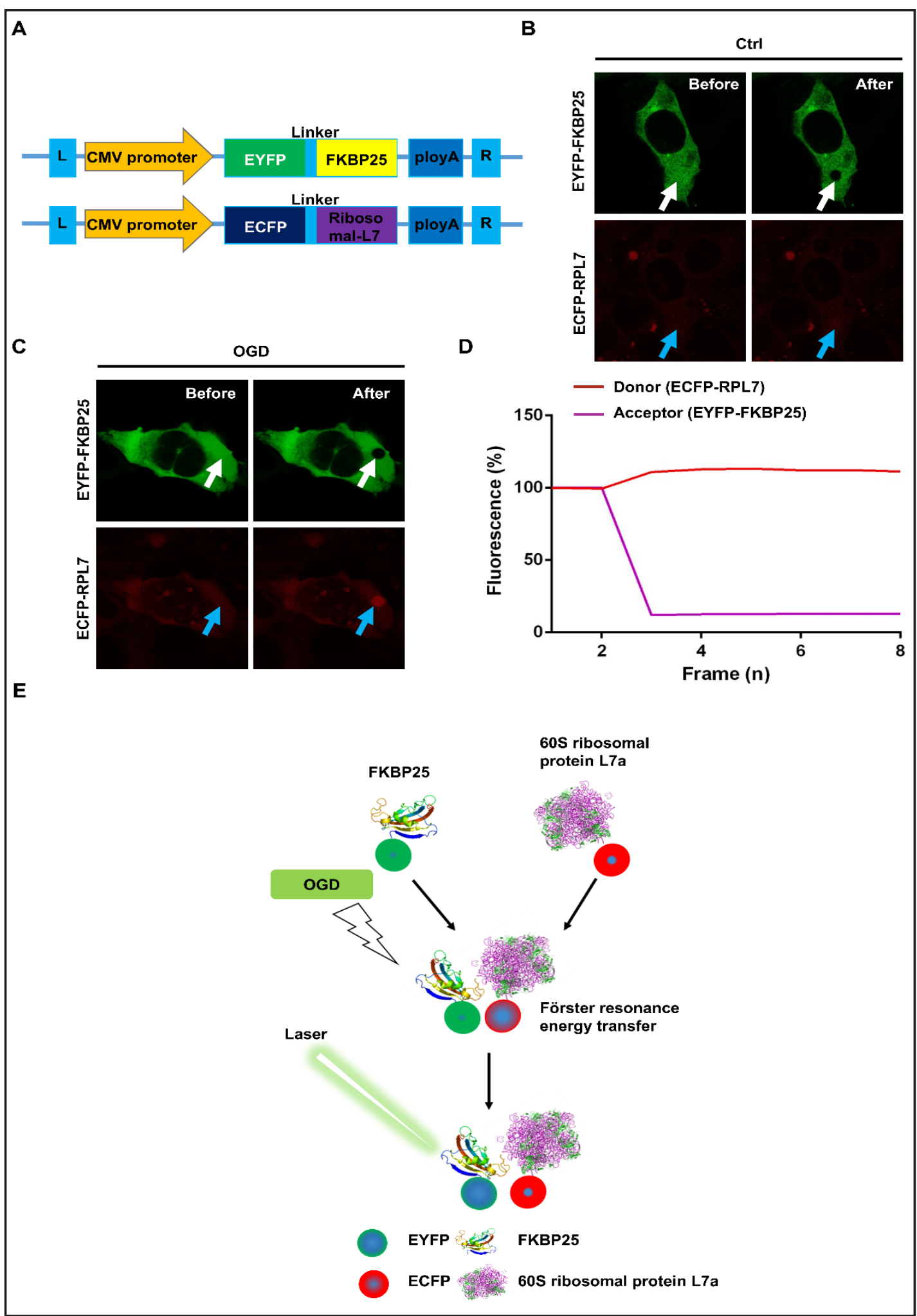

Fig. 7. FKBP25 binding with RPL7 was induced by OGD. (A) FKBP25 and RPL7 constructs used for FRET studies. Acceptor and donor fluorescence was recorded before and after photobleaching of whole cell under normal (B) or OGD treatment (C). (D) Fluorescence of acceptor and donor was recorded frame by frame as shown in (C). (E) Schematic diagrams of EYFP-FKBP25 binding with ECFP-RPL7 detected by FRET following OGD. 


\section{Cellular Physiology Cell Physiol Biochem 2018:47:2018-2030

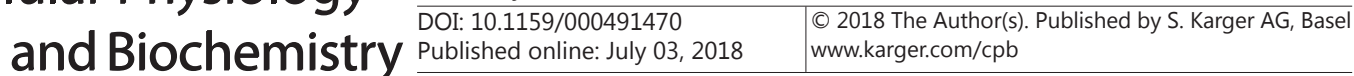

Fig. 8. Effect of FKBP25 nuclear translocation after OGD. (A) The effects of FKBP25 on PARP1 levels were examined in cell lysates of endothelial cells. Cells were transfected with FKBP25 lentivirus followed by $6 \mathrm{~h}$ of OGD or control stimulation. The proteins were immunoblotted with antibodies against FKBP25 and PARP1. (B) Quantitative analysis of protein levels for PARP-1 (89 kDa) following FKBP25 overexpression. Data are expressed as the densitometry ratio of the control (mean \pm S.E.M). ${ }^{* * *} \mathrm{P}<0.01$ versus control; ${ }^{\mathrm{P}}<0.05$; versus OGD.
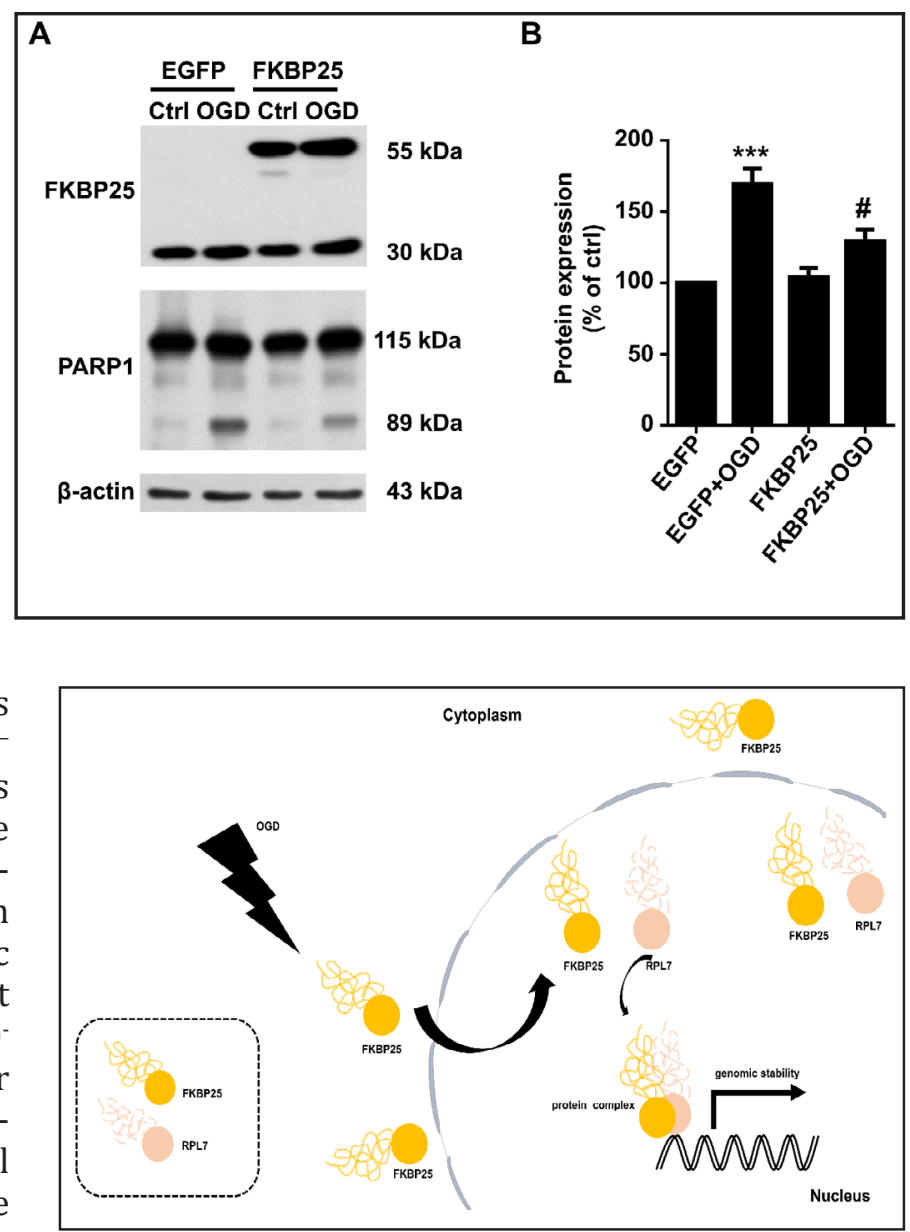

Fig. 9. Schematic illustration of the mechanisms by which the nuclear import of FKBP25 and binding with 60S ribosomal protein $\mathrm{L7a}$ are protective stress responses to ischemia/ nitrosative stress injury.

induced NO production and reduced endothelial dysfunction $[30,31]$. Therefore, we suggest the causal link between nitrosative stress and nuclear translocation of FKBP25 during ischemia.

A member of the FKBP family has been reported to be mediate nuclear translocation. For example, the FKBP52/FKBP51 expression ratio affects NF- $\mathrm{KB}$ nuclear translocation by forming complexes with NF- $\mathrm{KB}$ in HEK 293-T cells [32], and the nuclear localization of FKBP25 might affects various nuclear proteins for chromatin remodeling and packaging of DNA, in U937 cells [2]. Here, we found that the expression of truncated FKBP25 (1-108) was localized in the cytosol, which suggests that OGD-mediated downstream signaling other than the PPIase domain is involved in its nuclear translocation. We found that RPL7 interacted with FKBP25 by mass spectrometry analysis between control and OGD-treated endothelial cells. Our studies show that the fragment of 1-108 aa plays an important role in FKBP25 cytosolic localization, and ribosomal protein L7a contributes FKBP25 translocation from cytosol to the nucleus.

FK506 is considered a powerful neuroprotection mediator in focal and global cerebral ischemia through FKBP 12, 38 and 51 [7-10]. Stress-responsive FKBP51 regulates AKT2AS160 signaling and metabolic function [33]. Notably, the localization and intensity of FKBP12 increased in the cytosol and decreased in the nucleus until $24 \mathrm{~h}$ after reperfusion in hippocampus CA1 neurons [34]. PARP-1 was reported to be a key effector of cell death 
of neurovascular units in ischemic brain injury $[35,36]$. In the present study, we found that OGD-induced PARP-1 increasing was inhibited by nuclear FKBP25 overexpression in endothelial cells. Therefore, the FKBP25-60S ribosomal protein L7a complex strengthens FKBP25 nuclear translocation, which might plays a key role on FKBP25 protection of endothelial cell under ischemic stress (Fig. 9). These findings have been interpreted to mean that FKBP family proteins have distinct roles in different subtypes of physiological or pathological context $[15,37,38]$.

Our data provide a possible strategy for regulating the function of FKBP25, developing specific inhibitors of nitrosative stress. The current study has suggested that the $\mathrm{N}$-terminal helix-loop-helix domain and C-terminal FK506-binding domain interact with each other and that both domains are involved in FKBP25-DNA binding [3]. Further studies is essential for examining the molecular mechanisms of FKBP25 in the nucleus, especially functional significance of the FKBP25-60S ribosomal protein L7a in more detail, will provide a better understanding of the pathological events of stroke.

\section{Acknowledgements}

This work was supported in part by National Natural Science Foundations of China (81473202, 81673415 and 81460550), Zhejiang Provincial Natural Science Foundation of China (2017KY563), Hangzhou science and Technology Foundation of China (20172016A05) and the Zhejiang Province Program for Cultivation of High-level Health talents.

\section{Disclosure Statement}

No competing financial interests exist.

\section{References}

1 Ochocka AM, Kampanis P, Nicol S, Allende-Vega N, Cox M, Marcar L, Milne D, Fuller-Pace F, Meek D: FKBP25, a novel regulator of the p53 pathway, induces the degradation of MDM2 and activation of p53. FEBS Lett 2009;583:621-626.

2 Galat A, Thai R: Rapamycin-binding FKBP25 associates with diverse proteins that form large intracellular entities. Biochem Biophys Res Commun 2014;450:1255-1260.

3 Prakash A, Shin J, Rajan S, Yoon HS: Structural basis of nucleic acid recognition by FK506-binding protein 25 (FKBP25), a nuclear immunophilin. Nucleic Acids Res 2016;44:2909-2925.

4 Hamelberg D, Shen T, McCammon JA: Phosphorylation effects on cis/trans isomerization and the backbone conformation of serine-proline motifs: accelerated molecular dynamics analysis. J Am Chem Soc 2005;127:1969-1974.

5 Schwartz TU, Schmidt D, Brohawn SG, Blobel G: Homodimerization of the G protein SRbeta in the nucleotide-free state involves proline cis/trans isomerization in the switch II region. Proc Natl Acad Sci U S A 2006;103:6823-6828.

-6 Narimatsu Y, Kubota T, Furukawa S, Morii H, Narimatsu H, Yamasaki K: Effect of glycosylation on cis/trans isomerization of prolines in IgA1-hinge peptide. J Am Chem Soc 2010;132:5548-5549.

7 Morioka M, Fukunaga K, Kai Y, Todaka T, Yano S, Hamada J, Miyamoto E, Ushio Y: Intravenously injected FK506 failed to inhibit hippocampal calcineurin. Biochem Biophys Res Commun 2001;286:802-806.

8 Shioda N, Han F, Moriguchi S, Fukunaga K: Constitutively active calcineurin mediates delayed neuronal death through Fas-ligand expression via activation of NFAT and FKHR transcriptional activities in mouse brain ischemia. J Neurochem 2007;102:1506-1517.

-9 Brecht S, Schwarze K, Waetzig V, Christner C, Heiland S, Fischer G, Sartor K, Herdegen T: Changes in peptidyl-prolyl cis/trans isomerase activity and FK506 binding protein expression following neuroprotection by FK506 in the ischemic rat brain. Neuroscience 2003;120:1037-1048. 


\section{Cellular Physiology Cell Physiol Biochem 2018;47:2018-2030 \begin{tabular}{l|l} 
DOI: 10.1159/000491470 & $\begin{array}{l}\text { O 2018 The Author(s). Published by S. Karger AG, Basel } \\
\text { www.karger.com/cpb }\end{array}$
\end{tabular}}

10 Labrande C, Velly L, Canolle B, Guillet B, Masmejean F, Nieoullon A, Pisano P: Neuroprotective effects of tacrolimus (FK506) in a model of ischemic cortical cell cultures: role of glutamate uptake and FK506 binding protein $12 \mathrm{kDa}$. Neuroscience 2006;137:231-239.

11 Wei XE, Zhang FY, Wang K, Zhang QX, Rong LQ: Assembly of the FKBP51-PHLPP2-AKT signaling complex in cerebral ischemia/reperfusion injury in rats. Brain Res 2014;1566:60-68.

12 Edlich F, Weiwad M, Wildemann D, Jarczowski F, Kilka S, Moutty MC, Jahreis G, Lucke C, Schmidt W, Striggow F, Fischer G: The specific FKBP38 inhibitor N-(N',N'-dimethylcarboxamidomethyl)cycloheximide has potent neuroprotective and neurotrophic properties in brain ischemia. J Biol Chem 2006;281:1496114970.

13 Dilworth D, Upadhyay SK, Bonnafous P, Edoo AB, Bourbigot S, Pesek-Jardim F, Gudavicius G, Serpa JJ, Petrotchenko EV, Borchers CH, Nelson CJ, Mackereth CD: The basic tilted helix bundle domain of the prolyl isomerase FKBP25 is a novel double-stranded RNA binding module. Nucleic Acids Res 2017;45:1198912004.

14 Dilworth D, Gudavicius G, Xu X, Boyce A, O'Sullivan C, Serpa JJ, Bilenky M, Petrochenko EV, Borchers $\mathrm{CH}$, Hirst M, Swayne LA, Howard P, Nelson CJ: The prolyl isomerase FKBP25 regulates microtubule polymerization impacting cell cycle progression and genomic stability. Nucleic Acids Res 2018;46:24592478.

15 Gudavicius G, Dilworth D, Serpa JJ, Sessler N, Petrotchenko EV, Borchers CH, Nelson CJ: The prolyl isomerase, FKBP25, interacts with RNA-engaged nucleolin and the pre-60S ribosomal subunit. RNA 2014;20:1014-1022.

-16 Lopez E, Berna-Erro A, Salido GM, Rosado JA, Redondo PC: FKBP25 and FKBP38 regulate non-capacitative calcium entry through TRPC6. Biochim Biophys Acta 2015;1853:2684-2696.

17 Tao RR, Wang H, Hong LJ, Huang JY, Lu YM, Liao MH, Ye WF, Lu NN, Zhu DY, Huang Q Fukunaga K, Lou YJ, Shoji I, Wilcox CS, Lai EY, Han F: Nitrosative stress induces peroxiredoxin 1 ubiquitination during ischemic insult via E6AP activation in endothelial cells both in vitro and in vivo. Antioxid Redox Signal 2014;21:1-16.

-18 Wang CK, Ahmed MM, Jiang Q, Lu NN, Tan C, Gao YP, Mahmood Q, Chen DY, Fukunaga K, Li M, Chen Z, Wilcox CS, Lu YM, Qin ZH, Han F: Melatonin ameliorates hypoglycemic stress-induced brain endothelial tight junction injury by inhibiting protein nitration of TP53-induced glycolysis and apoptosis regulator. J Pineal Res 2017;63.

19 Tao RR, Huang JY, Shao XJ, Ye WF, Tian Y, Liao MH, Fukunaga K, Lou YJ, Han F, Lu YM: Ischemic injury promotes Keap1 nitration and disturbance of antioxidative responses in endothelial cells: a potential vasoprotective effect of melatonin. J Pineal Res 2013;54:271-281.

20 Jiang Q, Li XR, Wang CK, Cheng J, Tan C, Cui TT, Lu NN, James TD, Han F, Li X: A fluorescent peptidyl substrate for visualizing peptidyl-prolyl cis/trans isomerase activity in live cells. Chem Commun (Camb) 2018;54:1857-1860.

21 Wang H, Hong LJ, Huang JY, Jiang Q, Tao RR, Tan C, Lu NN, Wang CK, Ahmed MM, Lu YM, Liu ZR, Shi WX, Lai EY, Wilcox CS, Han F: P2RX7 sensitizes Mac-1/ICAM-1-dependent leukocyte-endothelial adhesion and promotes neurovascular injury during septic encephalopathy. Cell Res 2015;25:674-690.

-22 Liu Z, Han Y, Li L, Lu H, Meng G, Li X, Shirhan M, Peh MT, Xie L, Zhou S, Wang X, Chen Q, Dai W, Tan CH, Pan S, Moore PK, Ji Y: The hydrogen sulfide donor,GYY4137, exhibits anti-atherosclerotic activity in high fat fed apolipoprotein E(-/-) mice. Br J Pharmacol. 2013;169:1795-1809.

23 Hong LJ, Jiang Q, Long S, Wang H, Zhang LD, Tian Y, Wang CK, Cao JJ, Tao RR, Huang JY, Liao MH, Lu YM, Fukunaga K, Zhou NM, Han F: Valproic Acid Influences MTNR1A Intracellular Trafficking and Signaling in a beta-Arrestin 2-Dependent Manner. Mol Neurobiol 2016;53:1237-1246.

24 Jiang Q, Gao Y, Wang C, Tao R, Wu Y, Zhan K, Liao M, Lu N, Lu Y, Wilcox CS, Luo J, Jiang LH, Yang W, Han F: Nitration of TRPM2 as a Molecular Switch Induces Autophagy During Brain Pericyte Injury. Antioxid Redox Signal 2017;27:1297-1316.

25 Dembowski JA, Ramesh M, McManus CJ, Woolford JJ: Identification of the binding site of Rlp7 on assembling 60S ribosomal subunits in Saccharomyces cerevisiae. RNA 2013;19:1639-1647.

26 Tao RR, Ji YL, Lu YM, Fukunaga K, Han F: Targeting nitrosative stress for neurovascular protection: new implications in brain diseases. Curr Drug Targets 2012;13:272-284.

27 Casas AI, Geuss E, Kleikers P, Mencl S, Herrmann AM, Buendia I, Egea J, Meuth SG, Lopez MG, Kleinschnitz C, Schmidt H: NOX4-dependent neuronal autotoxicity and BBB breakdown explain the superior sensitivity of the brain to ischemic damage. Proc Natl Acad Sci U S A 2017;114:12315-12320. 


\section{Cellular Physiology Cell Physiol Biochem 2018;47:2018-2030 \begin{tabular}{l|l} 
DOI: 10.1159/000491470 & $\begin{array}{l}\text { O 2018 The Author(s). Published by S. Karger AG, Basel } \\
\text { www.karger.com/cpb }\end{array}$
\end{tabular}}

28 Wilcox CS, Pearlman A: Chemistry and antihypertensive effects of tempol and other nitroxides. Pharmacol Rev 2008;60:418-469.

29 Han F, Shirasaki Y, Fukunaga K: Microsphere embolism-induced endothelial nitric oxide synthase expression mediates disruption of the blood-brain barrier in rat brain. J Neurochem 2006;99:97-106.

-30 Long C, Cook LG, Hamilton SL, Wu GY, Mitchell BM: FK506 binding protein 12/12.6 depletion increases endothelial nitric oxide synthase threonine 495 phosphorylation and blood pressure. Hypertension 2007;49:569-576.

-31 Long C, Cook LG, Wu GY, Mitchell BM: Removal of FKBP12/12.6 from endothelial ryanodine receptors leads to an intracellular calcium leak and endothelial dysfunction. Arterioscler Thromb Vasc Biol 2007;27:15801586.

-32 Erlejman AG, De Leo SA, Mazaira GI, Molinari AM, Camisay MF, Fontana V, Cox MB, Piwien-Pilipuk G, Galigniana MD: NF-kappaB transcriptional activity is modulated by FK506-binding proteins FKBP51 and FKBP52: a role for peptidyl-prolyl isomerase activity. J Biol Chem 2014;289:26263-26276.

-33 Balsevich G, Hausl AS, Meyer CW, Karamihalev S, Feng X, Pohlmann ML, Dournes C, Uribe-Marino A, Santarelli S, Labermaier C, Hafner K, Mao T, Breitsamer M, Theodoropoulou M, Namendorf C, Uhr M, Paez-Pereda M, Winter G, Hausch F, Chen A, Tschop MH, Rein T, Gassen NC, Schmidt MV: Stress-responsive FKBP51 regulates AKT2-AS160 signaling and metabolic function. Nat Commun 2017;8:1725.

-34 Fumoto N, Nakatsuka H, Ohta S, Kumon Y, Ohnishi T: Hippocampal CA1 neuron survival and cytosolic FKBP12, the $12 \mathrm{kDa}$ FK506-binding protein, after ischemia and tacrolimus treatment in gerbils. Neurosci Lett 2003;339:219-222.

35 Moroni F, Chiarugi A: Post-ischemic brain damage: targeting PARP-1 within the ischemic neurovascular units as a realistic avenue to stroke treatment. FEBS J 2009;276:36-45.

-36 Zhang R, Tang S, Huang W, Liu X, Li G, Chi H, Zhu M, Tang J: Protection of the brain following cerebral ischemia through the attenuation of PARP-1-induced neurovascular unit damage in rats. Brain Res 2015;1624:9-18.

-37 Kumar V, Sabatini D, Pandey P, Gingras AC, Majumder PK, Kumar M, Yuan ZM, Carmichael G, Weichselbaum R, Sonenberg N, Kufe D, Kharbanda S: Regulation of the rapamycin and FKBP-target 1/mammalian target of rapamycin and cap-dependent initiation of translation by the c-Abl protein-tyrosine kinase. J Biol Chem 2000;275:10779-10787.

-38 Stocki P, Sawicki M, Mays CE, Hong SJ, Chapman DC, Westaway D, Williams DB: Inhibition of the FKBP family of peptidyl prolyl isomerases induces abortive translocation and degradation of the cellular prion protein. Mol Biol Cell 2016;27:757-767. 